\title{
KOEBE DOMAINS FOR CERTAIN FAMILIES
}

\author{
A. W. GOODMAN
}

\begin{abstract}
The Koebe domain of a family of functions, analytic in the unit disk $E$, is the largest domain that is contained in $f(E)$ for every function of the family. In this paper we review some families for which the Koebe domains are known, and find the Koebe domains for some new families.
\end{abstract}

1. Introduction. In this paper we are concerned with various families $\mathscr{F}$ of functions each regular in the unit disk $E:|z|<1$. The Koebe domain for the family $\mathscr{F}$ is denoted by $K(\mathscr{F})$ and by definition this is the largest domain that is contained in $f(E)$ for every function $f(z)$ in $\mathscr{F}$.

Some results are trivial consequences of classical theorems on univalent functions. For example if $\mathscr{F}$ is the family of normalized functions univalent in $E$, then the Koebe $1 / 4$-theorem tells us the $K(\mathscr{F})$ is the disk $|w|<1 / 4$. If $\mathscr{F}$ is the subset of convex univalent functions then $K(\mathscr{F})$ is the disk $|w|<1 / 2$. Other examples will be mentioned at the end of this paper.

2. Bounded functions. Let $\mathscr{B}(A)$ be the family of functions

$$
f(z)=A z+\sum_{n=2}^{\infty} a_{n} z^{n}, \quad 0<A \leqslant 1,
$$

that are regular and satisfy $|f(z)|<1$ in $E$. In 1929 Landau [8] determined $K(\Re(A))$. Here we obtain the same result, but our proof is somewhat different and slightly shorter. We first describe the extremal function in

LEMMA 1. The function

$$
F(z)=e^{B / 2} \frac{1-e^{-B z /(1-z)}}{e^{B}-e^{-B z /(1-z)}}, \quad B>0
$$

has the following properties in $E$ : it is regular, $|F(z)|<1$, it omits $e^{-B / 2}$, and if $f(z) \in \mathscr{B}(C), 0<C<1$, and also omits $e^{-B / 2}$, then $F^{-1}(f(z))$ is regular in $E$, and satisfies the conditions of Schwarz's lemma.

We observe that $F(z)$ can be put in the form

$$
F(z)=\frac{\sinh B z / 2(1-z)}{\sinh B / 2(1-z)}
$$

Presented to the Society, January 4, 1978 under the title Koebe domains for certain classes of functions; received by the editors January 10, 1978.

AMS (MOS) subject classifications (1970). Primary 30A26; Secondary 30A36, 30A42.

(C) 1979 American Mathematical Society 0002-9939/79/0000-0165/\$03.00 
Proof. We build $F(z)$ by a sequence of mappings. First $\eta=-B z /(1-z)$ carries $E$ into the half-plane $\operatorname{Re} \eta<B / 2$. Then $\zeta=e^{\eta}$ maps this domain onto a surface consisting of infinitely many disks $|\zeta|<R=e^{B / 2}$, tied with a logarithmic branch point at $\zeta=0$. The function $\zeta(z)$ omits $\zeta=0$, but assumes every other value in $|\zeta|<R$ infinitely often. Finally the function $w=R(1-\zeta) /\left(R^{2}-\zeta\right)$ carries the disk $|\zeta|<R$ onto $|w|<1$ and takes $\zeta=0$ into $w=1 / R$. Thus $w=F(z)$ has the first three properties mentioned in the lemma. To see that $F^{-1}(f(z))$ is regular in $E$ we observe that it is regular in a neighborhood of the origin. Further it can be continued analytically throughout $E$, because $F^{\prime}(z)$ is never zero in $E$ and the only boundary points of $F(E)$ are the points $|w|=1$, and the omitted value $R=e^{-B / 2}$.

THEOREM 1 (LANDAU [8]). Let $\delta=\rho(A)$ be the solution of the equation

$$
A=\frac{-2 \delta \ln \delta}{1-\delta^{2}}, \quad 0<A<1,
$$

with $0<\rho(A)<1$. Then the Koebe domain for the family $\mathscr{B}(A)$ is the disk $|w|<\rho(A)$.

Proof. We first remark that if $f(z) \in \mathscr{B}(A)$, then so also is $e^{i \alpha} f\left(e^{-i \alpha} z\right)$ for every real $\alpha$. Thus if $f(z)$ omits some $w_{0}$, then by a rotation there is another function in the class that omits $\delta=\left|w_{0}\right|$. It follows that if a class of functions is invariant under the rotation $e^{i \alpha} f\left(e^{-i \alpha} z\right)$, then the Koebe domain is a disk.

Suppose now that $f(z)$ is in $\mathscr{B}(A)$ and omits $\delta$ where we can assume that $1>\delta>0$. We define $B$ by $\delta=e^{-B / 2}$ and with this $B$ and the function $F$ defined by equation (2) we set

$$
\varphi(z)=F^{-1}[f(z)]=\frac{f^{\prime}(0)}{F^{\prime}(0)} z+\cdots=\frac{A\left(e^{B}-1\right)}{B e^{B / 2}} z+\cdots
$$

Then by Schwarz's lemma applied to $\varphi(z)$

$$
A\left(e^{B}-1\right) / B e^{B / 2} \leqslant 1
$$

with equality if and only if $\varphi(z)=z$. If we replace $e^{B / 2}$ by $\delta$ in (6) we obtain

$$
A \leqslant \frac{-2 \delta \ln \delta}{1-\delta^{2}} \text {. }
$$

Since the right side of (6) is a strictly increasing function of $\delta$ we must have $\delta \geqslant \rho(A)$. Hence the Koebe domain for $\mathscr{B}(A)$ is the disk $|\omega|<\rho(A)$.

To see that the right side of (4) (or (7)) is a strictly increasing function of $\delta$ in $(0,1)$ we set $\delta=(1-u) /(1+u)$ (see Landau [8, p. 620]). Then

$$
\begin{aligned}
-\frac{2 \delta \ln \delta}{1-\delta^{2}} & =\frac{1-u^{2}}{2 u} \ln \frac{1+u}{1-u}=\left(1-u^{2}\right) \sum_{n=0}^{\infty} \frac{u^{2 n}}{2 n+1} \\
& =1-\sum_{n=1}^{\infty} \frac{2 u^{2 n}}{4 n^{2}-1},
\end{aligned}
$$

which is strictly decreasing in $u$. 
It is natural to extend Theorem 1 to the family $\mathscr{B}_{k}(A)$ of functions

$$
f(z)=A z^{k}+\sum_{n=k+1}^{\infty} a_{n} z^{n}, \quad 0<A<1,
$$

that are regular and satisfy $|f(z)|<1$ in $E$, where $k$ is a positive integer. Indeed we have

THEOREM 2. The Koebe domain for the family $\mathscr{B}_{k}(A)$ is the disk $|w|<\rho(A)$.

We set $F_{k}(z)=F\left(z^{k}\right)$ where $F(z)$ is the function defined by equation (2). Since $F_{k}(z)$ omits $\delta=e^{-B / 2}$, this shows that the Koebe domain for $\mathscr{B}_{k}(A)$ cannot be larger than the disk $|w|<\rho(A)$. If we try to imitate the subordination argument used in the proof of Theorem 1 , we find that we must make the additional assumption that $f(z) / z^{k}$ has no zeros in $E$. However, by modifying the original Landau proof we can avoid this additional assumption.

Suppose that $f(z)$ is in $\mathscr{B}_{k}(A)$ and omits $\delta$, where without loss of generality we assume that $0<\delta<1$. If we set

$$
\begin{aligned}
g(z) & =\frac{\delta-f(z)}{1-\delta f(z)}=\left(\delta-A z^{k}-\cdots\right)\left(1+\delta A z^{k}+\cdots\right) \\
& =\delta-A\left(1-\delta^{2}\right) z^{k}+\cdots,
\end{aligned}
$$

then $|g(z)|<1$ and $g(z) \neq 0$ in $E$. Consequently

$$
\begin{aligned}
h(z) & =-\ln \frac{g(z)}{\delta}=-\ln \left(1-\frac{A\left(1-\delta^{2}\right)}{\delta} z^{k}+\cdots\right) \\
& =\frac{-A\left(1-\delta^{2}\right)}{\delta} z^{k}+\cdots
\end{aligned}
$$

is regular and $\operatorname{Re} h(z)>-\ln (1 / \delta)$ in $E$. When we apply the Carathéodory bound $\left|b_{n}\right| \leqslant 2$ for the coefficients $b_{n}$ of a function with positive real part, to

$$
\frac{h(z)+\ln (1 / \delta)}{\ln (1 / \delta)}=1-\frac{A\left(1-\delta^{2}\right)}{\delta \ln (1 / \delta)} z^{k}+\cdots
$$

we obtain inequality (7).

Let $\mathscr{B}_{k}(1, M)$ denote the family of functions

$$
f(z)=z^{k}+\sum_{n=k+1}^{\infty} a_{n} z^{n}
$$

that are regular and satisfy $|f(z)|<M$ in $E$, where $M \geqslant 1$. A trivial transformation applied to Theorem 2 gives

Corollary 1. The Koebe domain for the family $\mathscr{B}_{k}(1, M)$ is the disk $|w|<M \rho(1 / M)$. 
We observe that

$$
\lim _{M \rightarrow \infty} M \rho\left(\frac{1}{M}\right)=\lim _{A \rightarrow 0} \frac{\rho(A)}{A}=0 .
$$

This gives

COROLlaRY 2. Let $\mathbb{Q}_{k}$ be the set of all functions of the form (12) that are regular in $E$. Then the only point common to $f(E)$ for every $f(z)$ in $Q_{k}$ is the origin.

In other words the Koebe "domain" for $\mathbb{Q}_{k}$ consists of just one point, $w=0$.

3. Some related families. Let $\mathscr{P}_{k}(A)$ denote the family of functions of the form

$$
p(z)=1+A z^{k}+\sum_{n=k+1}^{\infty} a_{n} z^{n}, \quad 0<A<2,
$$

which are regular and satisfy $\operatorname{Re}(p(z))>0$ in $E$. We apply the transformation $p(z)=(1+f(z)) /(1-f(z))$ to the functions in $\mathscr{B}_{k}(A)$. Since a covered domain goes into a covered domain, and the family $\mathscr{B}_{k}(A / 2)$ goes into the family $\mathscr{P}_{k}(A)$ we have

THEOREM 3. Let $k$ be a positive integer and let $0<A<2$. The Koebe domain for the family $\mathscr{P}_{k}(A)$ is the open disk with center at the point $\left(1+\rho^{2}(A / 2)\right) /\left(1-\rho^{2}(A / 2)\right)$ and radius $2 \rho(A / 2) /\left(1-\rho^{2}(A / 2)\right)$.

If we apply the transformation $g(z)=2 \alpha f(z) /(1-f(z))$ to a function $f(z)$ in $\mathscr{B}_{k}(A / 2 \alpha)$ we find that

$$
g(z)=A z^{k}+\sum_{n=k+1}^{\infty} a_{n} z^{n}
$$

is regular and satisfies $\operatorname{Re} f(z)>-\alpha$ in $E$. This gives

THEOREM 4. Let $k$ be a positive integer and let $\alpha, A>0$, with $A<2 \alpha$. Let $\mathcal{H}_{k}(\alpha, A)$ be the family of functions of the form (14) which are regular and satisfy $\operatorname{Re} g(z)>-\alpha$ in $E$. Then the Koebe domain for this family is the open disk with center at the point $2 \alpha \rho^{2}(A / 2 \alpha) /\left(1-\rho^{2}(A / 2 \alpha)\right)$, and radius $2 \alpha \rho(A / 2 \alpha) /\left(1-\rho^{2}(A / 2 \alpha)\right)$.

For the next family, the method is the same but the computations are more involved. Let $\mathcal{H}_{k}(\alpha, \beta, A)$ be the family of functions $g(z)$ of the form (14) that are regular and satisfy $-\alpha<\operatorname{Re} g(z)<\beta$ in $E$. Here $\alpha, \beta, A>0$ and we must impose the condition

$$
A \leqslant \frac{2(\alpha+\beta)}{\pi} \cos \left(\frac{\alpha-\beta}{\alpha+\beta} \cdot \frac{\pi}{2}\right) \equiv A^{*},
$$


because if this condition is not satisfied the family is empty, as we will soon prove.

To obtain the Koebe domain for the family $\mathcal{H}_{k}(\alpha, \beta, A)$ we need the univalent function $H(z)$ that takes $E$ onto the domain $-\alpha<\operatorname{Re} w<\beta$ with $H(0)=0$ and $H^{\prime}(0)>0$. Since

$$
L(z)=\left(1-i e^{i \gamma} z\right) /\left(1+i e^{-i \gamma} z\right)
$$

maps $E$ onto the half-plane $-\pi / 2+\gamma<\arg w<\pi / 2+\gamma$, a brief computation shows that

$$
\begin{aligned}
H(z) & \equiv \frac{\alpha+\beta}{\pi} i \ln \frac{1-i e^{i \gamma} z}{1+i e^{-i \gamma} z}, \quad \gamma=\frac{\alpha-\beta}{\alpha+\beta} \cdot \frac{\pi}{2} \\
& =\frac{2(\alpha+\beta)}{\pi} \sum_{n=1}^{\infty}(-1)^{n+1}\left[\frac{\cos (2 n-1) \gamma}{2 n-1} z^{2 n-1}-\frac{\sin 2 n \gamma}{2 n} z^{2 n}\right]
\end{aligned}
$$

has exactly the properties we wish. Further

$$
H^{\prime}(0)=\frac{2(\alpha+\beta)}{\pi} \cos \left(\frac{\alpha-\beta}{\alpha+\beta} \cdot \frac{\pi}{2}\right)=A^{*}>0 .
$$

THEOREM 5. Let $k$ be a positive integer, let $\alpha, \beta, A>0$, and satisfy condition (15) with strict inequality. Let $B$ denote the unique positive root of

$$
A=\frac{2(\alpha+\beta)}{\pi} \cos \left(\frac{\alpha-\beta}{\alpha+\beta} \cdot \frac{\pi}{2}\right) \frac{B e^{B / 2}}{e^{B}-1} .
$$

Then the Koebe domain for the family $\mathcal{H}_{k}(\alpha, \beta, A)$ is the image of the disk $|z|<e^{-B / 2}$ under $H(z)$.

Proof. If $F(z)$ is the function defined by equation (2), then $e^{-i \theta} F\left(e^{i \theta} z^{k}\right)$ omits $e^{-i \theta} e^{-B / 2}$ in $E$. Since the composite function $h_{k}(z)=H\left(e^{-i \theta} F\left(e^{i \theta} z^{k}\right)\right)$ is in $\mathcal{H}_{k}(\alpha, \beta, A)$, this proves that the Koebe domain cannot be any larger than the one described in the theorem. Conversely if $f(z)=A z^{k}+\ldots$ is in $\mathcal{H}_{k}(\alpha, \beta, A)$ then $H^{-1}(f(z))$ is bounded in $E$ and the image of $E$ under this function must cover the disk $|w|<e^{-B / 2}$.

The condition $A \leqslant A^{*}$ is necessary. Suppose that $f(z)$ is in $\mathcal{H}_{k}(\alpha, \beta, A)$ with $A>A^{*}>0$. Then $H^{-1}(f(z))=b z^{k}+\ldots$ satisfies the conditions of Schwarz's lemma and $b>1$. This is a contradiction. If $A=A^{*}$ then $f(z) \equiv$ $H\left(z^{k}\right)$ and the Koebe domain is the strip $-\alpha<\operatorname{Re} w<\beta$.

4. A family of univalent functions. Let $\mathcal{S H}(\alpha, \beta, A)$ be the family of functions $f(z)=A z+\ldots$ which are regular and univalent and satisfy $-\alpha<\operatorname{Re} f(z)<\beta$ in $E$. Here $\alpha, \beta, A>0$ and satisfy condition (15). To obtain the Koebe domain for this family we combine $H(z)$ with the extremal function $B(z)$ for bounded univalent functions.

Pick's Theorem [13]. The Koebe domain for the family of functions $f(z)=z$ $+\ldots$ that are regular, univalent, and satisfy $|f(z)|<M$ in $E$ is the disk 


$$
|w|<M(2 M-1)-2 M \sqrt{M(M-1)} \equiv R_{P}(M) .
$$

There is a univalent function $B(z)$ that maps $E$ onto the disk $|w|<M$ with a slit from $R_{P}$ to $M$ on the real axis. Further $B(0)=0$ and $B^{\prime}(0)=1$.

Now let $f(z)$ belong to $\mathcal{S} \mathcal{H}(\alpha, \beta, A)$. If $H(z)$ is the function defined by equation (16), then

$$
b(z) \equiv H^{-1}(f(z))=\frac{A}{A^{*}} z+\cdots
$$

is regular, univalent, and satisfies $|b(z)|<1$ in $E$. If we apply Pick's Theorem to $A^{*} A^{-1} b(z)$ we find that this function maps $E$ onto a domain that covers the disk $|w|<R_{P}\left(A^{*} / A\right)$. Consequently $f(z)=H(b(z))$ maps $E$ onto a domain that covers the image of the disk

$$
|z|<\frac{A}{A^{*}} R_{P}\left(\frac{A^{*}}{A}\right)=\frac{1}{A}\left(2 A^{*}-A-\sqrt{A^{*}\left(A^{*}-A\right)}\right)
$$

under $H(z)$. If $M \equiv A^{*} / A \geqslant 1$, then $H\left(e^{i \theta} B\left(e^{-i \theta} z\right) / M\right)$ is in $\mathcal{S} \mathcal{H}(\alpha, \beta, A)$ for all real $\theta$. This gives

THEOREM 6. The Koebe domain for the family $\mathcal{S} \mathcal{H}(\alpha, \beta, A)$ is the image of the disk (19) under $H(z)$ defined by (16), and $A^{*}$ is defined by (15).

We can let $\beta \rightarrow \infty$ in Theorem 6 , but it is simpler to proceed directly. Let $\delta \mathcal{H}(\alpha, A)$ be the family of functions $f(z)=z+\ldots$ that are regular, univalent, and satisfy $\operatorname{Re} f(z)>-\alpha$ in $E$. If we replace $H(z)$ by the function $H_{1}(z)=2 \alpha z /(1-z)$ and $A^{*}$ by $2 \alpha$, and repeat the above considerations we obtain

TheOREM 7. If $A \leqslant 2 \alpha$, the Koebe domain for the family $\mathcal{S} \mathcal{H}(\alpha, A)$ is the image of the disk $|w|<A^{-1}(4 \alpha-A-\sqrt{2 \alpha(2 \alpha-A)})$ under $H_{1}(z)=$ $2 \alpha z /(1-z)$.

5. A survey of known Koebe domains. Rogosinski [15, Theorem 11] found $K$, the Koebe domain for the family of normalized starlike univalent functions with fixed $\left|a_{2}\right|$. Netanyahu [12] found $K$ for the same extended family when the condition starlike is dropped. Pinchuk [14, Theorem 6.2] found $K$ for the family $V_{k}$ of functions of bounded boundary rotation. Goodman [3] found $K$ for the family of typically-real functions. Goodman and Saff [4] found $K$ for the family of functions convex in the direction of the imaginary axis. Miller [11] found $K$ for the family of alpha-starlike functions, and Coonce and Miller [2] extended the result to the subclass of functions that are $p$-fold symmetric. We have already mentioned Landau's result [8] for the family of bounded functions, and Pick's Theorem for bounded univalent function. The Koebe domain for normalized univalent functions with $p$-fold symmetry follows immediately from the Koebe 1/4-theorem.

We turn to the family of normalized univalent functions for which all the coefficients are real and some of its subsets. The entire family has been 
considered by Jenkins [5]. McGregor [10] found $K$ for the following three subsets (A) convex functions, (B) starlike functions and (C) functions convex in the direction of the imaginary axis. Krzyż and Reade [6] found $K$ for the following five subsets (A) circularly symmetric functions, (B) the intersection of $f(E)$ with the upper half-plane is convex in the direction of $e^{i \pi \alpha}(0 \leqslant \alpha \leqslant$ 1), (C) close-to-convex functions (D) odd convex functions, and (E) odd starlike functions.

We can also impose a Montel type normalization. This means that for some fixed $r_{0}$ with $0<r_{0}<1$, we consider the family of functions $f(z)$ regular and univalent in $E$ with $f(0)=0$ and $f\left(r_{0}\right)=r_{0}$. Lewandowski, Miazga and Szynal [9] found $K$ for the following three subsets (A) starlike with real coefficients, (B) convex with real coefficients, and (C) convex in the direction of the imaginary axis with real coefficients.

Kuz'mina [7] has considered families of univalent functions that omit two fixed values. The Koebe domain for univalent functions convex in the direction of the imaginary axis was found earlier by Reade and Ztotkiewicz (On univalent functions with two preassigned values, Proc. Amer. Math. Soc. 30 (1971), pp. 539-544), but the Goodman-Saff proof [4] is shorter.

ADDED IN PROOF. Let $\delta_{k}$ be the family of normalized univalent functions for which $f(E)$ is bounded by a quasi-conformal $k$-circle. Blevins found $K$ for $\delta_{k}$, see Canad. J. Math. 28 (1976), 627-631.

In a recent conversation, $\mathrm{E}$. Merkes asked for the Koebe domain for a normalized odd typically-real function. Apparently this problem is difficult because the extremal function is not $\sqrt{H\left(z^{2}\right)}$ where $H(z)$ is the extremal function in the family of typically-real functions used in [3].

\section{REFERENCES}

1. S. D. Bernardi, Bibliography of schlicht functions, Part I (1966), Part II (1977), Courant Institute of Mathematical Sciences, New York University.

2. H. B. Coonce and Sanford Miller, Distortion properties of p-fold symmetric alpha-starlike functions, Proc. Amer. Math. Soc. 44 (1974), 336-340.

3. A. W. Goodman, The domain covered by a typically-real function, Proc. Amer. Math. Soc. 64 (1977), 233-237.

4. A. W. Goodman and E. B. Saff, On univalent functions convex in one direction, Proc. Amer. Math. Soc. (to appear).

5. J. A. Jenkins, On univalent functions with real coefficients, Ann. of Math. 71 (1960), 1-15.

6. Jan Krzyż and Maxwell Reade, Koebe domains for certain classes of analytic functions, J. Analyse Math. 18 (1967), 185-195.

7. G. V. Kuz'mina, Covering theorems for functions holomorphic and univalent in a disk, Soviet Math. Dokl. 6 (1965), 21-25.

8. Edmund Landau, Uber die Blochsche Konstante und zwei verwandte Weltkonstanten, Math. Z. 30 (1929), 608-634.

9. Z. Lewandowski, J. Miazga and J. Szynal, Koebe domains for univalent functions with real coefficients under Montel's normalization, Ann. Polon. Math. 30 (1975), 333-336.

10. M. T. McGregor, On three classes of univalent functions with real coefficients, J. London Math. Soc. 39 (1964), 43-50.

11. Sanford Miller, Distortion properties of alpha-starlike functions, Proc. Amer. Math. Soc. 38 (1973), 311-318. 
12. Elisha Netanyahu, On univalent functions in the unit disk whose image contains a given disk, J. Analyse Math. 23 (1970), 305-322.

13. G. Pick, Über die konforme Abbildung eines Kreises auf ein schlichtes und zugleich beschränktes Gebiet, Akad. Wiss. Sitz. Vienna Math. Natur. Kl. 126 (1917), 247-263.

14. Bernard Pinchuk, Functions of bounded boundary rotation, Israel J. Math. 10 (1971), 6-16.

15. Werner Rogosinski, On subordinate functions, Proc. Cambridge Philos. Soc. 35 (1939), 1-26.

Department of Mathematics, University of SOUth Florida, Tampa, Florida 33620 